\title{
F Genetic Susceptibility to Infectious Diseases in the Qatari Population
}

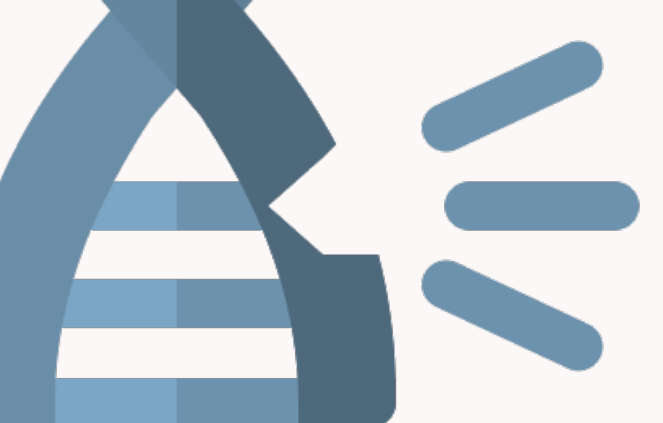

Maria K. Smatti ${ }^{1,2}$, Yasser A. Al-Sarraj2 ${ }^{2}$, Omar Albagha ${ }^{2,3^{*}}$, Hadi M. Yassine $\mathrm{e}^{1,2^{*}}$

${ }^{1}$ Biomedical Research Center, ${ }^{2}$ Hamad Bin Khalifa University, ${ }^{3}$ University of Edinburgh

\section{Background}

Infectious diseases (IDs) account for 8\% of deaths annually in Qatar, and therefore, represent a significant challenge for public health. Interestingly, the spread and severity of viral infections vary considerably between individuals and populations. The most recent example is SARS-CoV-2, which ranges from mild/asymptomatic illness in $80 \%$ of patients to a severe respiratory syndrome in $20 \%$. It has been previously reported that polymorphisms in genes linked to immunity can influence individuals' responses to infections as observed in tuberculosis, influenza, and HIV; however, studies exploring causal host genetic variants in IDs are still limited and dramatically skewed with regard to population inclusion. In fact, the genetic susceptibility to IDs in the Qatari population is largely unknown. Importantly, Qatari population represent five different ancestries, all of which are under-represented in the global genome databases.

\section{Methodology}
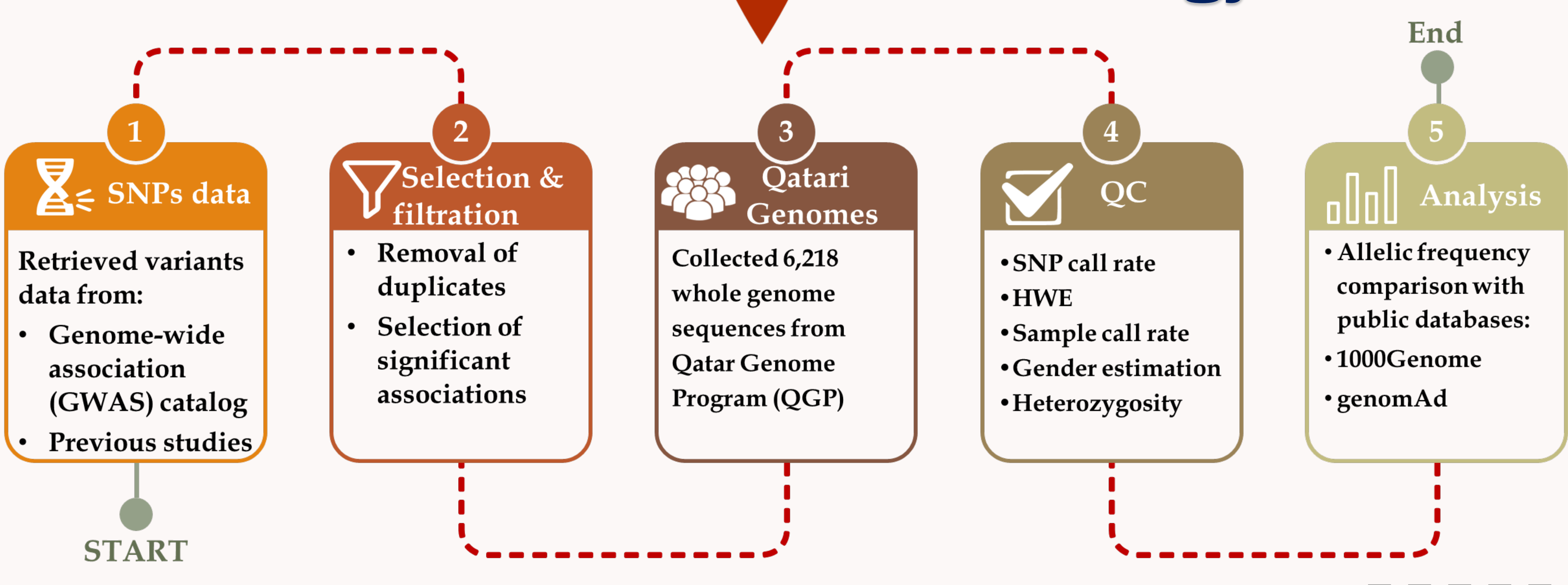

\section{Findings}

1- Summary of sequences/SNPs included in the study

\begin{tabular}{|ll|}
\hline Total number of QGP sequences & $\mathbf{6 , 2 1 8}$ \\
\hline Sequences passing QC & $\mathbf{6 , 0 4 7}$ \\
\hline $\begin{array}{l}\text { Total number of variants from GWAS } \\
\text { catalog }\end{array}$ & $\mathbf{2 , 2 5 8}$ \\
\hline Variants curated from QGP & $\mathbf{2 , 0 2 7}$ \\
\hline
\end{tabular}

2- Summary of infection-related SNPs in the GWAS catalog

$60 \%$

\begin{tabular}{|c|c|}
\hline $\begin{array}{l}\text { HIV } \\
\text { Hepatitis C }\end{array}$ & $\begin{array}{l}\text { Hepatitis B } \\
\text { Childhood ear infection }\end{array}$ \\
\hline Epstien Barr virus & - Tuberculosis \\
\hline$\square$ Herpes & Clostridium difficile \\
\hline — Influenza & - Rubella \\
\hline — Bacterial meningitis & a Gastrointestinal infection \\
\hline — Cytomegalovirus & $\llbracket$ Human papillomavirus \\
\hline Chlamydia & - Toxoplasma \\
\hline$=$ COVID & Malaria \\
\hline Fungal infections & Common infections \\
\hline
\end{tabular}

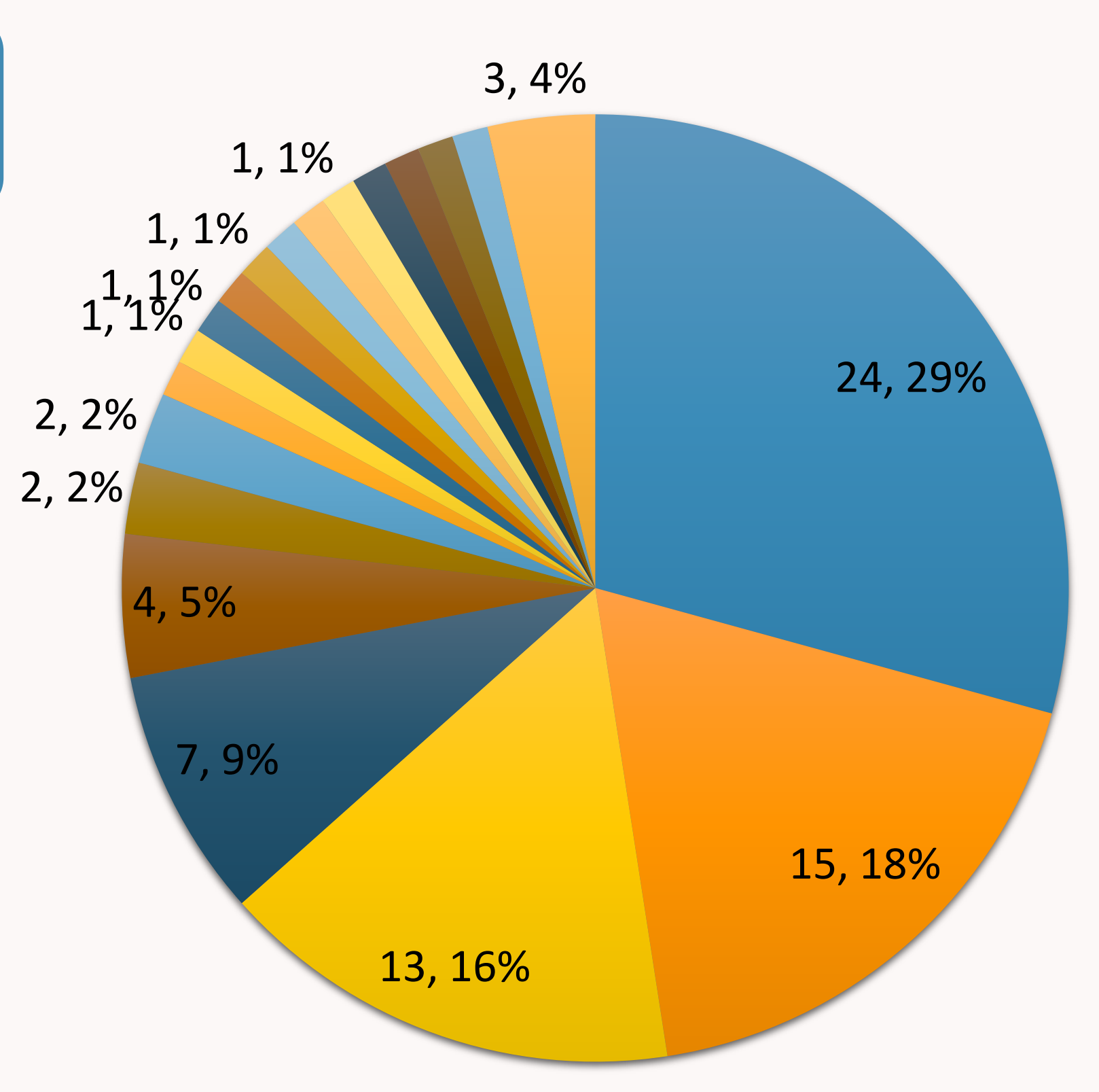

4- Fold difference of AF in QGP data compared to 1000 Genome data

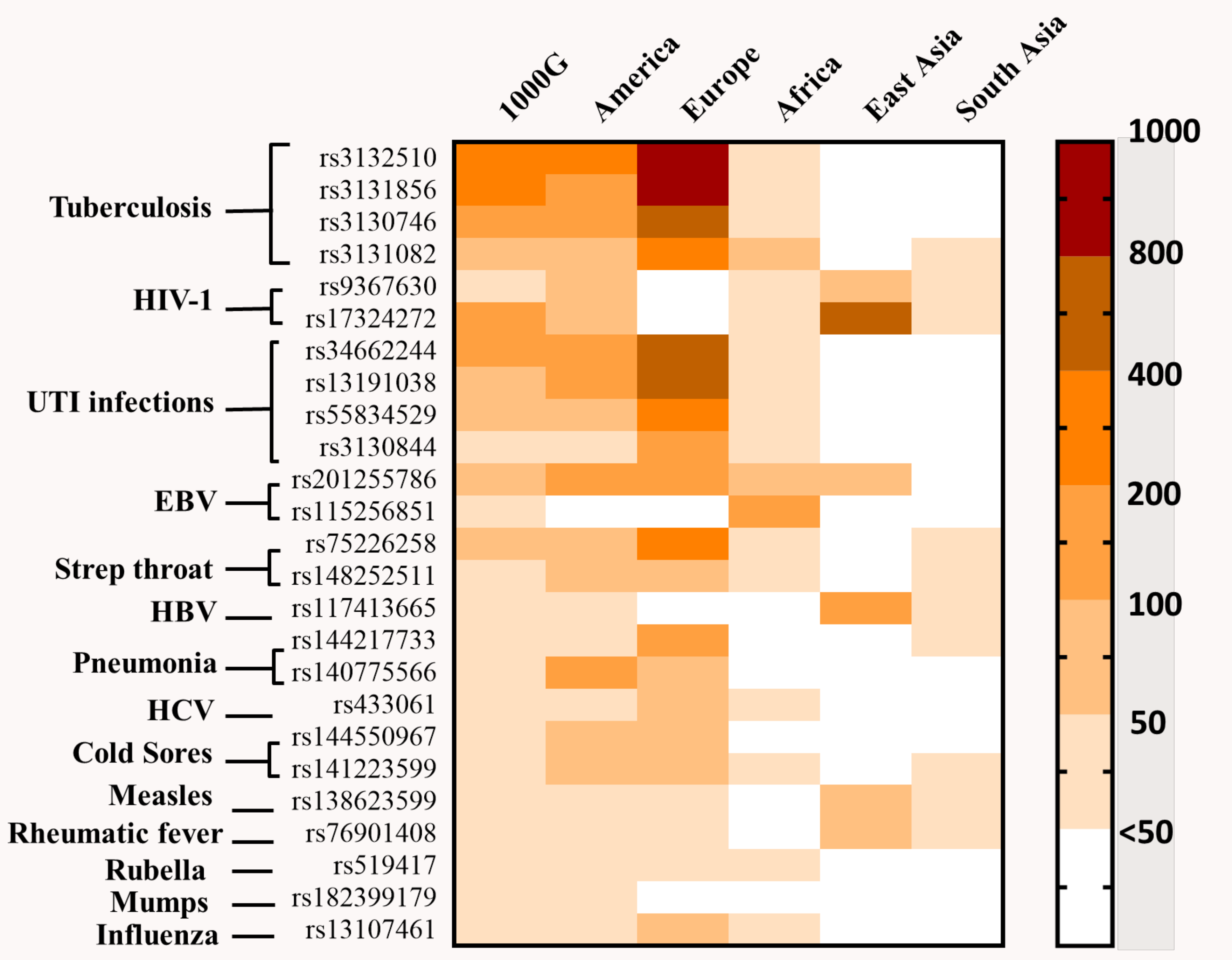

3- Allelic frequencies (AF) of infection-related SNPs in Qatari population compared to other populations

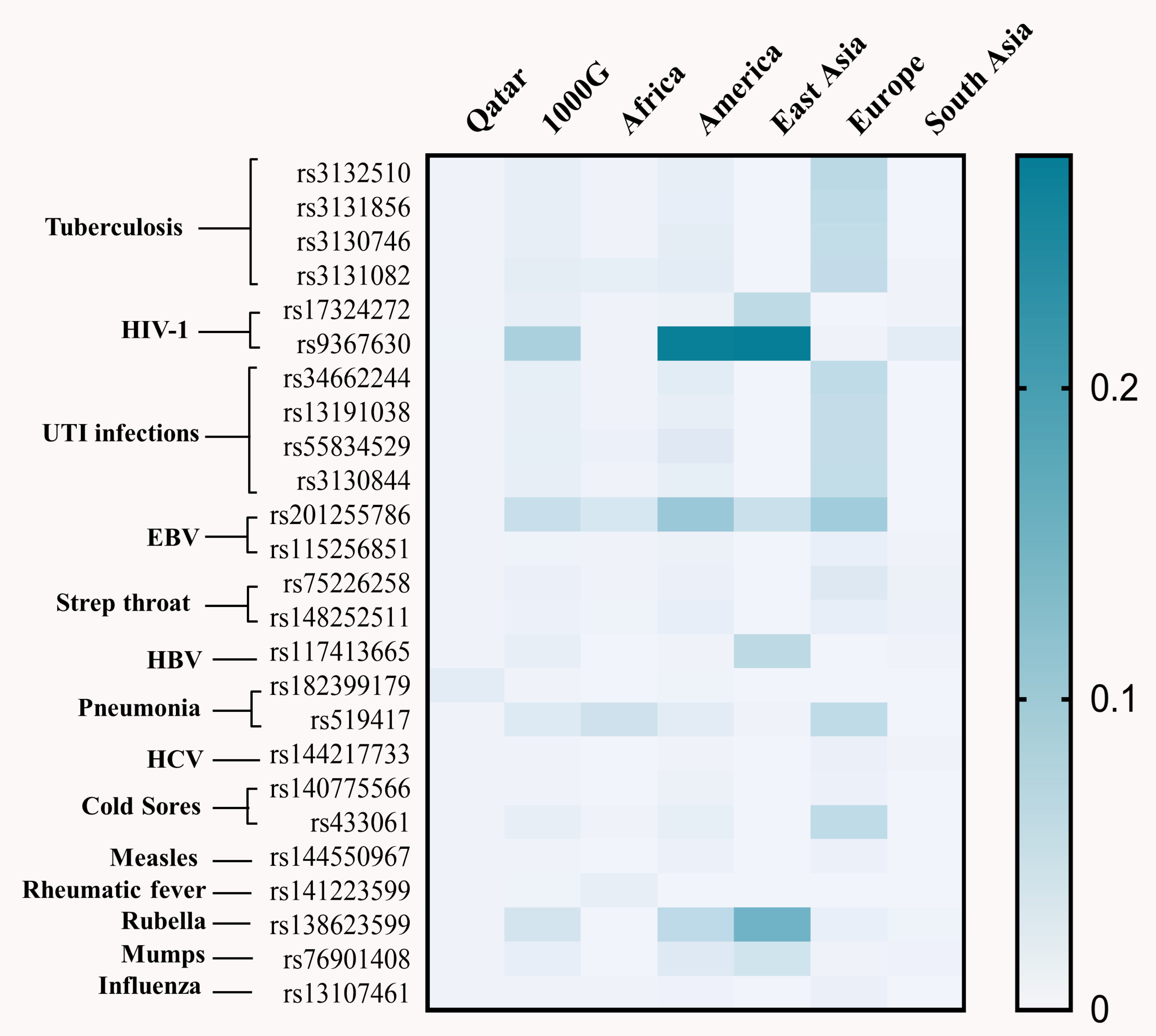

\section{Conclusions}

$>$ Host genetic variants that could influence the susceptibility and/or response to infectious diseases are distributed differently across populations.

$>$ The frequency of infections-related SNPs in the Qatari population was significantly lower for most infections.

> Most variants (78\%) showed negative fold change in the Qatari genomes.

> The most significant differences were observed in genes related to TB and HIV (200-940 and 160-710 fold change, respectively).

$>$ Although this report showed a lower susceptibility of the Qatari population to IDs in general, it might also indicate the presence of unknown variants that require further investigation in future GWAS. 